\title{
Adaptive Transmission-Reception-Sensing Strategy for Cognitive Radios with Full-duplex Capabilities
}

\author{
Wessam Afifi and Marwan Krunz \\ Department of Electrical and Computer Engineering, University of Arizona, Tucson, USA \\ E-mail: \{wessamafifi, krunz $\} @$ email.arizona.edu
}

\begin{abstract}
In this paper, we exploit recent advances in fullduplex (FD) communications and self-interference suppression (SIS) to improve the performance of an opportunistic spectrum access (OSA) system. Specifically, we consider secondary users (SUs) that are equipped with SIS-capable radios. These radios can operate in a simultaneous transmission-and-sensing (TS) mode to improve the detection probability of primary users (PUs), or in a simultaneous transmission-and-reception (TR) mode to enhance the SU throughput. The radios can also revert to the standard sensing-only (SO) mode or perform channel switching (CS). The competing goals of the full-duplex TS and TR modes give rise to a spectrum-awareness/efficiency tradeoff, which can be optimized by allowing the $\mathrm{SU}$ link to adaptively switch between various modes, depending on the forecasted PU dynamics. In practice, SIS is imperfect, resulting in residual self-interference that degrades the sensing performance in the TS mode. Accordingly, we adopt a waveform-based sensing approach, which allows an SU to detect (with high accuracy) the PU signal in the presence of self-interference (and noise). In such a context, we analyze the sensing performance in the TS mode by deriving the false-alarm and detection probabilities. We also derive the throughput and the PU-SU collision probability for the TS and TR modes, which we then use to establish an optimal mode-selection strategy that maximizes an SU utility function subject to a constraint on the PU collision probability. This utility rewards the SU instantly for successful communication (throughput), but also includes a longterm component that depends on the outcomes of the action taken by the SU (the selected mode from the set $\{$ TR, TS, SO, CS $\}$ ). Our results show that the proposed adaptive strategy results in about $50 \%$ reduction in the collision probability and twice the throughput of the half-duplex case. The results also indicate that the SU should operate in the TR mode if it has a high belief regarding the PU idleness over a given channel. As this belief decreases, the SU should switch to the TS mode to monitor any change in the PU activity while transmitting. At very low belief values, where the PU is highly likely to be active, the SU should switch to another channel.
\end{abstract}

Keywords-Self-interference cancellation, full-duplex communications, opportunistic spectrum access, cognitive radios, spectrum awareness/efficiency tradeoff.

\section{INTRODUCTION}

Until recently, the idea of simultaneous transmission and reception over the same channel (STAR-S) was not deemed possible. The reason is that while a wireless device is receiving data, its own transmission produces strong self-interference, which makes the decoding process impossible. One way to solve this problem is to suppress the node's self-interference. However, traditional self-interference suppression (SIS) techniques (e.g., RF and digital interference cancellation) have not been sufficient to suppress the self-interference signal so that STAR-S can take place. Even simultaneous transmission and reception over different frequencies (STAR-D) is not straightforward, particularly when the transmit and receive bands are not sufficiently separated (in practice, filters are not ideal, and sidelobes/spectral leakage is deemed to occur). In this paper, we focus on STAR-S (the more challenging case), which we simply refer to as full-duplex (FD) communication.

By combining novel and traditional SIS techniques, the authors in [1]-[5] have demonstrated the feasibility of FD communication. In [1], the authors proposed an antennabased SIS technique in which two properly placed transmit antennas and one receive antenna are used to nullify selfinterference at the receiving antenna. This technique has three main drawbacks. First, it generates additional interference in the far field, i.e., it increases the interference at the receivers of other nodes in the neighborhood. Second, it has a bandwidth limitation, as antenna placement is determined by a single carrier frequency. However, wireless transmissions typically involve a band of frequencies. Third, the peer node may fall into a deep fading region due to the appearance of destructive interference points in the far field. These concerns were addressed in [2], where the authors used only two antennas and proposed an interference cancellation mechanism based on signal inversion. Recently, the authors in [6] proposed an FD system for $802.11 \mathrm{ac}$ devices using only one antenna. The main objective of these works is to bring down a node's selfinterference to the noise level. As an example, a WiFi device has to suppress around $110 \mathrm{~dB}$ of its own transmitted signal (assuming a transmit power of $20 \mathrm{dBm}$ ) to reduce it to the noise level [6].

In parallel with the developments in SIS techniques, there have been a number of works that exploit SIS/FD capabilities in network-protocol design, in the contexts of MIMO communications [7]-[10] and dynamic spectrum access (DSA) [11], [12]. Assuming multiple antennas per node, the authors in [10] addressed the issue of choosing between MIMO and FD, as both need multiple antennas. They showed that the optimal strategy is a combination of both schemes. In [13] the authors studied power control in wireless FD devices with imperfect SIS. They developed an optimal dynamic power allocation scheme that maximizes the sum-rate of a number of users.

In this paper, we consider a DSA system, where secondary users (SUs) have imperfect SIS capabilities, allowing them to suppress a fraction of their self-interference. This partial SIS capability can be exploited to support simultaneous transmission-and-sensing (TS) by the SU so as to reduce the collision probability with primary users (PUs), or simultaneous transmission-and-reception (TR) to enhance the SU throughput. The ability to operate in either mode gives rise to a 
spectrum awareness/efficiency tradeoff. More specifically, an SU may improve the spectrum utilization by operating in the TR mode, which will dramatically increase the throughput of the SU link. On the other hand, the SU may exploit its SIS capabilities in the TS mode, enabling it to monitor the PU activity while transmitting and to quickly vacate the channel whenever such activity is detected. This motivates the need for an "optimal" transmission-sensing-reception strategy introduced in this paper.

In a practical DSA system, SUs may have to switch to a different operation mode rather than the TS and TR modes to either avoid collision with PUs or to exploit another opportunity on a different channel. Specifically, since the sensing efficiency in the TS mode decreases as the SIS efficiency decreases, in some cases the SU needs to operate in a sensingonly (SO) mode to achieve an acceptable sensing outcome. Also, having a relatively high belief that the PU is active may return a high collision probability in the TS/TR modes. In that case, the SU should stop transmission and just monitors the channel. Considering the availability of multiple idle channels, an SU may decide to perform channel switching (CS) if the $\mathrm{PU}$ is more likely to return to the current operational channel.

An important aspect of the system design is to determine the strategy followed by SUs to adaptively switch between different modes (TR, TS, SO, and CS), considering the highly dynamic spectrum environment and the possibility of colliding with PUs. Our objective is to find the optimal strategy that maximizes the SU's utility (e.g., goodput) under a constraint on the PU collision probability. This strategy is found to be threshold-based, with thresholds that depend on the SU's belief about the PU's state. Based on this belief, the SU will take an optimal action and then update this belief according to the outcome of the action taken. The outcome is ACK/NACK in case of a transmission decision, free/busy in case of a sensing decision, and decoded/undecoded in case of reception. The SU may also get a combination of these outcomes in the TR and TS modes.

The problem of finding the optimal access strategy at an SU device has been studied before [14]-[17], but for halfduplex (HD) devices. In [14], the authors considered the quickest detection problem of the PU idle period when multiple PUs are present. In their scheme, the SU chooses an action from the following: spectrum sensing, channel switching, or data transmission. The authors in [15] studied the sensingthroughput tradeoff and proposed a scheme in which the SU can have multiple consecutive sensing or transmission periods, determined according to the SU's belief about the state of the PU. In their scheme, the SU has only two options: spectrum sensing or data transmission. The objective was to maximize the SU's utility, which rewards the SU for successful transmission and penalizes it for collisions. Another adaptive scheme was proposed in [17], where a secondary transmitter adapts its sensing and transmission durations according to its belief regarding the PU state of activity. In this case, the SU can either stay idle, sense the spectrum, or transmit its data. The motivation behind the "staying idle" action was to save energy when the probability that the PU is idle is very low.

In [11], we proposed applying SIS/FD in DSA systems and introduced the TR and TS modes. However, our treatment was limited to energy-based spectrum sensing (for the TS mode).
Energy detection cannot differentiate between a PU signal and a residual self-interference signal. Hence, it is inefficient under low SIS capabilities. This problem is unlikely to happen in waveform-based sensing, whereby the sensed waveform is contrasted with well-known patterns (pilots, preambles, etc.) of the PU signal. In [11], we also studied the traditional sensingthroughput tradeoff for the TR and TS modes and determined the optimal sensing and transmission durations for the SU that maximizes its throughput subject to a constraint on the SU/PU collision probability. However, this paper is different because in [11], we only considered the TR and TS modes. Unlike this paper, we have not discussed in [11] the belief update process, the outcomes of different modes, the utility formulation, or the derivations of the optimal strategy.

The contributions of this paper are as follows. First, we consider a DSA system where SUs are partially capable of SIS. We analyze the waveform-based spectrum sensing technique for the TS mode, which is crucial especially at imperfect SIS, and derive the false-alarm and detection probabilities. Second, we derive the probability of successful transmission for the SU, its achievable throughput, and the PU collision probability in both TS and TR modes, taking into consideration that SIS may be imperfect and assuming different channel conditions at the communicating SUs. Third, we propose an optimal adaptive strategy at the SU for switching between the TR, TS, SO, and CS modes. The criteria for choosing the optimal action is to maximize the SU's utility subject to a constraint on the PU collision probability. To achieve this goal, we formulate the problem as a partially observable decision process and analyze the four actions by formulating the myopic and long-term rewards. To the best of our knowledge, this is the first paper to address the optimal transmission-reception-sensing strategy for SUs with imperfect FD/SIS capabilities.

The rest of the paper is organized as follows. We describe the system model in Section II. In Section III, we derive the false-alarm and detection probabilities under waveform-based sensing for the TS mode and compare them with the HD case. We formulate the SU decision process and obtain the optimal adaptive SU spectrum access strategy in Section IV. Finally, we present our numerical results and conclude the paper in Sections V and VI, respectively.

\section{SySteM MODEL}

As shown in Figure 1, we consider a DSA network, where SUs are opportunistically operating on the licensed PUs channels. PUs can access the available channels at will, and are not aware of the SU's presence. The PU activity is modeled as an alternating ON/OFF random process. Let the OFF and ON durations be denoted by $X$ and $Y$, with corresponding probability distributions $f_{X}$ and $f_{Y}$, and means $\bar{X}$ and $\bar{Y}$, respectively. These distributions are assumed to be independent and can be constructed at the SU through measurements [18], [19].

Each secondary device is capable of partial or complete SIS, enabling it to operate in the TS and TR modes, along with the SO and CS modes. We use $\chi_{i}$ to quantify the SIS capability of the $i$ th SU, $\chi_{i} \in[0,1]$. Specifically, $\chi_{i}$ is the ratio between the residual self-interference signal and the original one. If $\chi_{i}=0$, the node can totally suppress the self-interference 


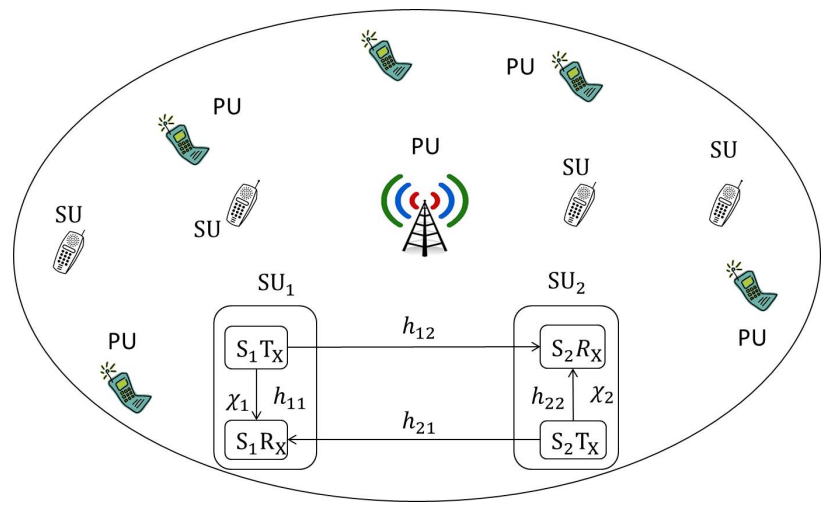

Fig. 1. System model of our DSA network, where SUs are equipped with SIS/FD capabilities and opportunistically access the spectrum of PUs. An $\mathrm{SU}_{i}$ consists of a transceiver $\left(\mathrm{S}_{i} \mathrm{~T}_{X}\right.$ acts as a transmitter, while $\mathrm{S}_{i} \mathrm{R}_{X}$ is the receiver) with a given SIS capability factor $\chi_{i}$.

signal (i.e., perfect SIS); otherwise, it can only suppress a fraction $1-\chi_{i}$ of its self-interference (i.e., imperfect SIS). As an example, for a residual self-interference signal of power (in watts) equal to $1 \%$ of the power of the original self-interference signal, this translates into $\chi_{i}=\sqrt{0.01}=0.1 . \chi_{i}$ may differ from one node to another, depending on the employed SIS technique.

We assume that at a given time instant, and a given frequency, only one SU link is active in a given geographical area. Hence, we focus on the case where different SU links cannot interfere with each other, for example, by implementing an appropriate channel access scheme. Existing techniques can be used to tackle the issue of secondary-secondary interference (see [20], for example), and will not be addressed here. Let $P_{i}$ and $\sigma_{i}^{2}$ denote the transmission power and noise variance at node $i$, and let $h_{i j}$ be the channel gain between transmitter $i$ and receiver $j$. Although the opportunistic spectrum consists of multiple channels, the SU can only monitor/operate on one channel at a time. Sensing multiple channels has already been discussed in several papers, and can be easily incorporated [21].

To sum up how our system works, consider the secondary link shown in Figure 1, which consists of two nodes, $\mathrm{SU}_{1}$ and $\mathrm{SU}_{2} . \mathrm{SU}_{1}$, for example, starts its communication with its peer with an initial belief value. Depending on the adaptive access strategy, the SU chooses the optimal action that maximizes its utility while maintaining a certain QoS threshold for the PU communication. The action's outcome may be ACK/NACK in case of transmission, free/busy in case of sensing, and decoded/undecoded in case of reception. The SU may also get a combination of these outcomes in the TR and TS modes. Depending on these outcomes, the SU updates its belief about the PU state. Getting an ACK/free/decoded outcome will increase the SU's belief that the PU is idle with a certain degree. However, getting a NACK/busy/undecoded outcome will increase the SU's belief that the PU is busy. Based on this belief, and according to our spectrum access strategy, the SU will take the optimal action, and so on.

\section{A. SU Operation Modes}

1) TS mode: Using SIS techniques, the SU can carry out the spectrum sensing process while transmitting its data. This has two advantages over the Listen-Before-Talk (LBT) scheme. First, from the SU's perspective, transmitting while sensing increases the SU throughput, and reduces the frequency of interrupting its transmission (such interruptions are detrimental to any real-time communications). Second, the SU can monitor the PU activity while transmitting. Hence, a better PU detection performance is achieved. This parallel sensing process may be done over multiple (consecutive) short periods instead of one long sensing period. To do that, the SU performs $m$ sensing actions $T_{S i}, i=1,2, \ldots, m$, while transmitting data for a period of $T$ seconds (see Figure 2(a)). The motivation behind this approach is to account for the tradeoff between sensing efficiency and the timeliness in detecting PU activity. On the one hand, increasing the sensing duration improves the sensing efficiency. However, such an increase implies delaying the time to make a decision regarding the change of PU activity. Thus, in the TS mode, we have a total of $m$ sensing durations. If at the end of any given sensing period, PU activity is detected, the SU aborts its current transmission and updates its belief to determine the next action. We use the term FD sensing to refer to the sensing process in the TS mode. Note that under imperfect SIS, such sensing has worse performance than the traditional SO mode due to the residual self-interference signal.

2) TR mode: In the TR mode, the SU transmits and receives data simultaneously over the same channel, as shown in Figure 2(b). Denote the transmission and reception durations by $T$ and $T_{R}$, respectively. For simplicity, we assume that $T_{R}=T$. Although operating in the TR mode enhances the SU's throughput, the SU will not be able to monitor the PU state. Hence, the probability of colliding with the PU will be higher than that of the TS mode.

3) SO mode: In this mode, the SU senses the spectrum for a duration $T_{S}$, which we refer to as $H D$ sensing. Under imperfect SIS, the TS mode is not always efficient. Hence, the SU may switch to the SO mode to get more accurate sensing results.

4) CS mode: The SU may switch to another channel and carry out spectrum sensing on this new channel if it believes that the PU is very likely to return to the currently used channel. Existing techniques can be used to select the channel sensing order (see [22], for example). However, any previous information about the new channel that was obtained from prior sensing attempts are discarded.

Although we will not consider the transmission-only (TO) mode as an option, we will use it for comparison purposes. The reason for not considering it is that the sensing cost is almost negligible. Hence, there is no advantage of the TO mode over the TS mode.

\section{WAVEFORM-BASED FD SENSING}

A significant amount of DSA literature has focused on energy-based sensing. Despite its simplicity, this technique cannot differentiate between different types of users. In the TS mode, residual self-interference can cause energy detection 
PU dynamics

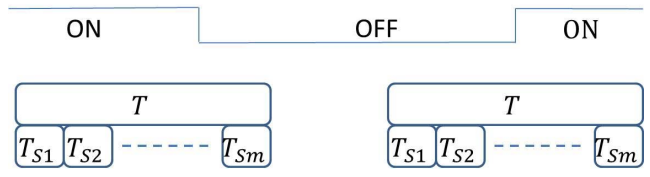

(a) Transmission-Sensing (TS) mode

\begin{tabular}{|c|}
\hline$T$ \\
\hline$T_{R}$ \\
$T$ \\
$T_{R}$ \\
\hline
\end{tabular}

(b) Transmission-Reception (TR) mode

Fig. 2. FD operation modes for the SU.

to wrongly indicate PU activity. In this paper, we study the sensing performance of the TS mode, assuming waveformbased sensing.

Waveform-based sensing utilizes known patterns in the PU signal, such as preambles and pilot symbols. These patterns are typically used for channel estimation, synchronization, equalization, etc. To detect the presence of the PU signal, waveformbased sensing correlates a known pattern with the received signal [23], [24]. In this section, we analyze waveform-based sensing under FD operation and derive the false-alarm and detection probabilities for the SU, assuming a given SIS factor $\chi$.

In the TS mode, the hypothesis test of whether the channel is occupied by a PU or not can be formulated as follows:

$$
r(n)= \begin{cases}\chi s(n)+w(n) & H_{0} \text { (if PU is idle) (1a) } \\ l(n)+\chi s(n)+w(n) & H_{1} \text { (if PU is busy) (1b) }\end{cases}
$$

where $r(n)$ is the $n$th sample of the discretized received signal, $s(n)$ is the self-interfering SU signal, $l(n)$ is the received PU signal, and $w(n)$ is the additive white Gaussian noise with variance $\sigma_{w}^{2}$. We assume that $s(n)$ is a zero-mean complex random signal with variance $\sigma_{s}^{2}$. We also assume that all signal samples are independent, hence $r(n)$ 's are also independent.

In the case of HD sensing, where no self-interference is present, the hypothesis test can be written as:

$$
\tilde{r}(n)= \begin{cases}w(n) & H_{0} \text { (if PU is idle) } \\ l(n)+w(n) & H_{1} \text { (if PU is busy) }\end{cases}
$$

where $\tilde{r}(n)$ is the $n$th sample of the received signal in the HD case.

The performance of any sensing technique is measured by the false-alarm probability $\left(P_{f}\right)$ and the detection probability $\left(P_{d}\right) . P_{f}$ and $P_{d}$ are defined as the probabilities that the SU declares the sensed channel to be busy given hypothesis $H_{0}$ and $H_{1}$, respectively. A good system should have high $P_{d}$ to reduce collisions between SUs and PUs. At the same time, a lower $P_{f}$ value results in a higher SU throughput due to a reduction in the missed transmission opportunities.

The decision metric, denoted by $M$, in waveform-based sensing is based on correlating the received samples $r(n)$ 's with known pattern samples, and then comparing $M$ against a given threshold $\gamma$ to determine the state of the sensed channel.
Formally, $M$ is given by:

$$
M=\operatorname{Re}\left[\sum_{n=1}^{N} r(n) l^{*}(n)\right]
$$

where $l^{*}(n)$ is the conjugate of $l(n), N$ is the number of samples, and Re is the real value. Substituting (1a) and (1b) into (3), we obtain $M$ under hypothesis $H_{0}$ and $H_{1}$, denoted by $M_{0}$ and $M_{1}$, respectively:

$$
\begin{gathered}
M_{0}=\operatorname{Re}\left[\sum_{n=1}^{N}\left(\chi s(n) l^{*}(n)+w(n) l^{*}(n)\right)\right] \\
M_{1}=\sum_{n=1}^{N}|l(n)|^{2}+\operatorname{Re}\left[\sum_{n=1}^{N}\left(\chi s(n) l^{*}(n)+w(n) l^{*}(n)\right)\right] .
\end{gathered}
$$

For FD sensing, $P_{f}$ and $P_{d}$ are given by:

$$
\begin{aligned}
& P_{f}=\operatorname{Pr}\left[M_{0}>\gamma\right]=1-F_{M_{0}}(\gamma) \\
& P_{d}=\operatorname{Pr}\left[M_{1}>\gamma\right]=1-F_{M_{1}}(\gamma)
\end{aligned}
$$

where $F_{M_{0}}(\gamma)$ and $F_{M_{1}}(\gamma)$ are the CDFs of the random variables $M_{0}$ and $M_{1}$, respectively.

Proposition 1: Using the central limit theorem (for a large $N$ ), the pdf of $M_{0}$ can be approximated by a Gaussian distribution with mean $\mu_{M_{0}}=0$ and the following variance:

$$
\sigma_{M_{0}}^{2}=\frac{N}{2}\left[\chi^{2} \mathrm{E}|s(n)|^{2} \mathrm{E}|l(n)|^{2}+\mathrm{E}|w(n)|^{2} \mathrm{E}|l(n)|^{2}\right] \text {. }
$$

Hence, the false-alarm probability can be written as:

$$
P_{f}=Q\left(\frac{\gamma-\mu_{M_{0}}}{\sigma_{M_{0}}}\right)
$$

where $Q$ is the complementary distribution function of a standard Gaussian random variable. Substituting for $\mu_{M_{0}}$ and $\sigma_{M_{0}}^{2}$ in (9), we get the false-alarm probability for FD sensing as follows:

$$
P_{f}=Q\left(\frac{\gamma}{\chi^{2} \sigma_{s}^{2}+\sigma_{w}^{2}} \sqrt{\frac{2}{N S N R^{(F D)}}}\right)
$$

where $S N R^{(F D)}$ is the SNR at the secondary receiver while sensing the spectrum in the FD case and is given by:

$$
S N R^{(F D)}=\frac{\mathrm{E}|l(n)|^{2}}{\chi^{2} \mathrm{E}|s(n)|^{2}+\mathrm{E}|w(n)|^{2}} .
$$

Note that $S N R^{(F D)}$ contains the self-interference term, in addition to noise. Furthermore, the number of samples $N$ can be described as a function of the sensing duration $T_{S i}$, $i=1,2, \ldots, m$, and the sampling rate $f_{S}$ as follows:

$$
N=T_{S i} f_{S} .
$$

Proposition 2: For a large $N$, the pdf of $M_{1}$ can be approximated by a Gaussian distribution with mean $\mu_{M_{1}}=$ 
$N \mathrm{E}|l(n)|^{2}$ and the following variance

$$
\begin{aligned}
\sigma_{M_{1}}^{2} & =N\left[\mathrm{E}|l(n)|^{4}-\mathrm{E}^{2}|l(n)|^{2}\right. \\
& \left.+\frac{1}{2}\left(\chi^{2} \mathrm{E}|s(n)|^{2} \mathrm{E}|l(n)|^{2}+\mathrm{E}|w(n)|^{2} \mathrm{E}|l(n)|^{2}\right)\right] .
\end{aligned}
$$

The proof for these two propositions are omitted for brevity, but can be found online in our technical report [25].

The detection probability for the waveform-based FD sensing can be written as follows:

$$
P_{d}=Q\left(\frac{\gamma-\mu_{M_{1}}}{\sigma_{M_{1}}}\right)
$$

Substituting for $\mu_{M_{1}}$ and $\sigma_{M_{1}}^{2}$ in (13), we get:

$$
P_{d}=Q\left(\frac{\gamma /\left(\chi^{2} \sigma_{s}^{2}+\sigma_{w}^{2}\right)-N S N R^{(F D)}}{\sqrt{N\left[(\alpha-1)\left(S N R^{(F D)}\right)^{2}+S N R^{(F D)} / 2\right]}}\right)
$$

where $\alpha$ is a parameter of the PU signal that is related to its randomness [23]. As an example $\alpha=2$ for complex Gaussian signals and can range from 1 to 2 for other signal types. Formally, $\alpha$ is defined as follows:

$$
\alpha \stackrel{\text { def }}{=} \frac{\mathrm{E}|l(n)|^{4}}{\mathrm{E}^{2}|l(n)|^{2}} .
$$

The false-alarm and detection probabilities in (10) and (14) for FD sensing converge to HD sensing at perfect SIS (i.e., $\chi=0$ ), as shown in the following equations for a specific sensing duration $T_{S i}, i=1,2, \ldots, m$ :

$$
\begin{gathered}
\tilde{P}_{f}=Q\left(\frac{\gamma}{\sigma_{w}^{2}} \sqrt{\frac{2}{N S N R^{(H D)}}}\right) \\
\tilde{P}_{d}=Q\left(\frac{\gamma /\left(\sigma_{w}^{2}\right)-N S N R^{(H D)}}{\sqrt{N\left[(\alpha-1)\left(S N R^{(H D)}\right)^{2}+S N R^{(H D)} / 2\right]}}\right)
\end{gathered}
$$

where $\tilde{P}_{f}$ and $\tilde{P}_{d}$ are the false-alarm and detection probabilities for HD sensing, respectively, and $S N R^{(H D)}$ is the SNR at the secondary receiver while sensing the spectrum in the HD case:

$$
S N R^{(H D)}=\frac{\mathrm{E}|l(n)|^{2}}{\mathrm{E}|w(n)|^{2}} .
$$

$P_{f}$ and $P_{d}$ derived in (10) and (14) for FD sensing are functions of the sensing threshold $\gamma$. The optimal sensing threshold $\gamma^{*}$ can be determined according to the system requirements on $P_{f}$ and $\left(1-P_{d}\right)$. For a target $P_{f}$ or $P_{d}, \gamma^{*}$ can be calculated by finding the inverse of the $Q$-functions in (10) and (14), respectively. As an example, for a system with a requirement that $P_{f}$ and $\left(1-P_{d}\right)$ are equal. The optimal sensing threshold $\gamma^{*}$ can be determined by equating $P_{f}$ with
$1-P_{d}$ in (9) and (13), resulting in:

$$
\gamma^{*}=\frac{\mu_{M_{0}} \sigma_{M_{1}}+\mu_{M_{1}} \sigma_{M_{0}}}{\sigma_{M_{0}}+\sigma_{M_{1}}} .
$$

Substituting this $\gamma^{*}$ in (9) and (13), and after some mathematical manipulations, we obtain the following for $P_{f}$ and $P_{d}$ :

$$
\begin{gathered}
P_{f}=Q\left(\frac{\sqrt{N S N R^{(F D)}}}{\sqrt{(\alpha-1) S N R^{(F D)}+1 / 2}+\sqrt{1 / 2}}\right) \\
P_{d}=1-Q\left(\frac{\sqrt{N S N R^{(F D)}}}{\sqrt{(\alpha-1) S N R^{(F D)}+1 / 2}+\sqrt{1 / 2}}\right) .
\end{gathered}
$$

\section{OPTIMAL SU STRATEGY}

In this section, we present an optimal strategy for operating an FD-capable SU link.

\section{A. Problem Formulation}

To optimize the selection of the operational mode at an SU, we formulate the problem as a partially observable decision process. Let $S=\{0,1\}$ be the state space, which defines the actual state (idle or busy) of the channel currently being observed by the SU. The action space at an SU is given by $A=$ $\{T R, T S, S O, C S\}$. While observing the PU channel, the SU has to choose an action from the set $A$. The outcome/observation space for the SU depends on the action taken. Since the TR action consists of two simultaneous processes (transmission and reception), there exist two outcomes for each of these processes. Specifically, for the reception part, the SU will observe the outcome $\{D\}$, which means that the SU was able to decode the received message, or the outcome $\{U\}$, which stands for undecoded message. For the transmission part of the TR mode, the SU may get ACK or a NACK from the peer SU, which are denoted by $\{A\}$ and $\{N\}$, respectively. Similarly, a TS action consists of two simultaneous processes (transmission and sensing). The SU will also observe two outcomes for the sensing process: ( $\{F\}$ for free or $\{B\}$ for busy). The outcomes of the transmission part of the TS mode are similar to that of the TR action. Finally, the observed outcomes for the SO/CS actions are $\{F\}$ or $\{B\}$. Altogether, these various actions result in an observation space $O=\{D, U, A, N, F, B\}$. Later on, we present a reward function, which maps the state and action space to a reward value.

Our objective is to let the SU choose actions sequentially in time so as to maximize the expected reward over some random finite horizon. This can be done using stochastic dynamic programming. It is known that the sufficient statistics for choosing the optimal action at each time $t$ is the belief [26], which is defined as the a posteriori probability $p_{t} \in[0,1]$ that the PU is idle at time $t$ given the observation history. We consider a similar setup as in [15] for the partially observable decision process part, where the time index $t$ is defined as the time elapsed since the PU has switched from ON to OFF. Hence, $t=0$ is the start of the PU idle period (with some negligible error compared to the actual start of the PU idle period), which is assumed to be known to the SU, and therefore 

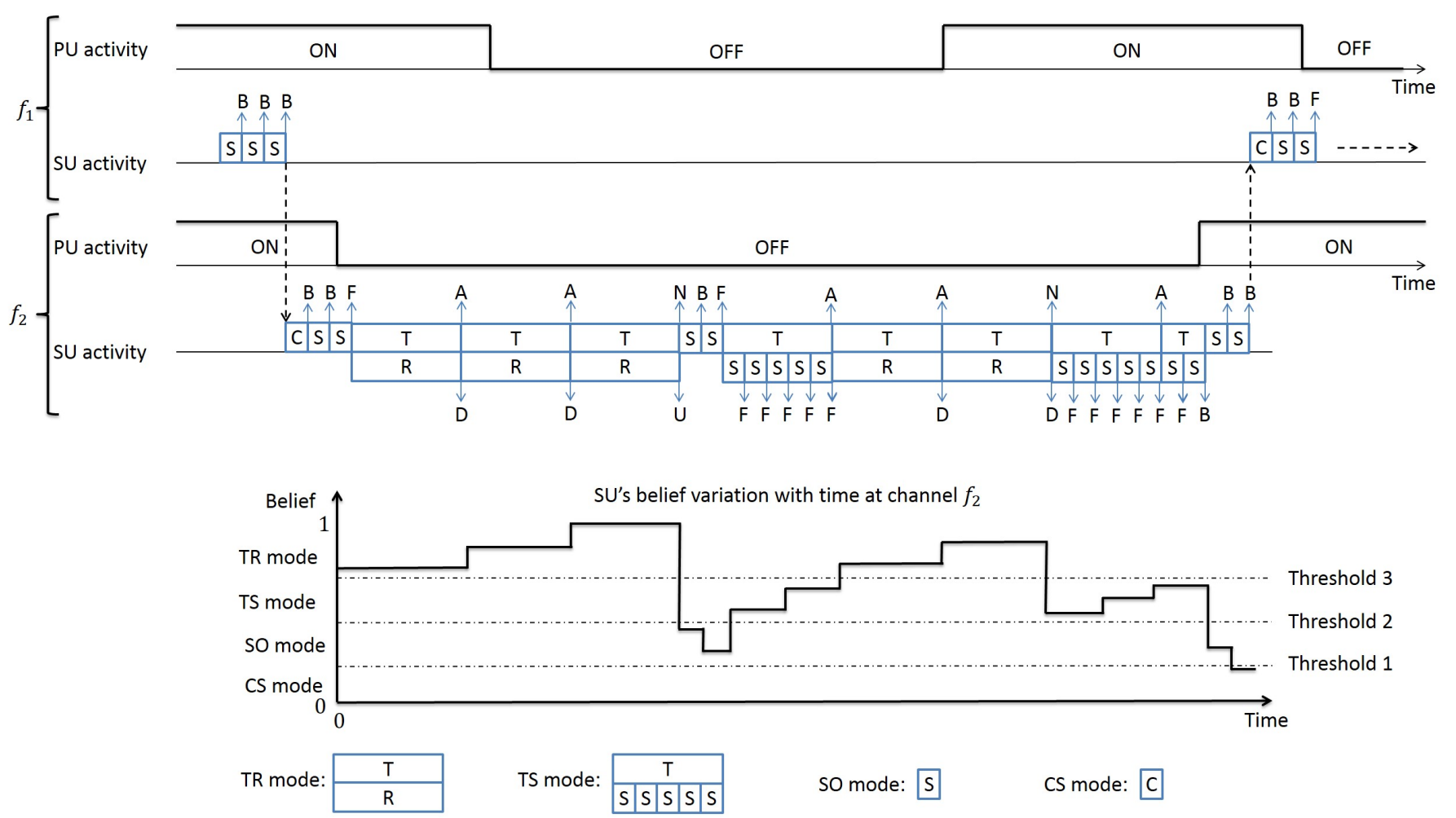

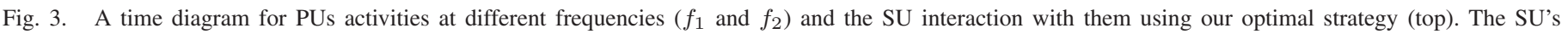

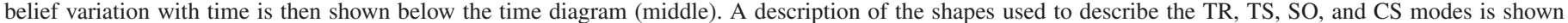

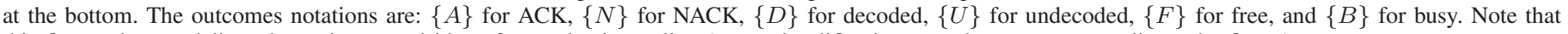
this figure aims to deliver the main general idea of our adaptive policy (some simplifications are done to not complicate the figure).

$p_{0}=1-\tilde{P}_{f} \cdot{ }^{1}$ Starting from $t=0$, the SU keeps tracking of time, and applying the optimal mode selection policy until switching to a new channel (CS action). At this time, the $\mathrm{SU}$ resets the algorithm and keeps sensing/switching between different channels until catching the start of the PU idle period. Once the beginning of an idle period is detected, the SU sets its timer to $t=0$, and then starts applying the optimal policy. While deriving our optimal policy, we assume that both communicating SUs always have data to transmit. However, at the end of this section, we will discuss the more general case that accounts for the traffic flow between different SUs.

Figure 3 shows a simplified example to illustrate how the SU will adaptively choose its optimal actions and update its belief according to the resulting outcomes. In this example, we have two channels $\left(f_{1}\right.$ and $\left.f_{2}\right)$ occupied with PUs, and an SU link, where two SUs are trying to opportunistically communicate on one of these channels. Assume that the SU starts monitoring channel $f_{1}$, which is happened to be busy. After getting multiple busy outcomes, it decides to switch to another channel $f_{2}$, which is sensed free after multiple busy outcomes. At this point the SU starts its timer (i.e., $t=0)$. In that case, the belief happens to be larger than a certain threshold (say, threshold 3), then the SU starts with the TR mode. The outcomes of the first TR action are ACK and decoded. Hence, as shown in the figure, the SU's belief increases and recommends the TR mode again. However, it

\footnotetext{
${ }^{1}$ As an example, the SU may keep sensing a busy channel in an HD fashion until it gets a free outcome. In that case the initial belief $p_{0}=1-\tilde{P}_{f}$.
}

happened that the SU receives a NACK and undecoded for the third TR action (may be for a deep fading at this time instance). The SU then updates its belief, which decreases below threshold 2, which implies that the SU should sense the spectrum (i.e., SO mode). The SU keeps sensing until the belief goes over threshold 2. In that case, the SU switches to the TS mode and updates its belief according to the outcomes that it gets. The SU continues switching between different operation modes and updates its belief accordingly as shown in Figure 3 until its belief goes below threshold 1. In that case the SU switches to another channel, and so on. Note that the PU has returned to channel $f_{2}$, while the SU is operating on the same channel. However, it happened that the SU is in the TS mode, which makes the SU detect the PU activity while transmitting. In that case the SU stops its transmission quickly to prevent collision with the PU (see Figure 3 above).

Following any given action $a \in A$ and depending on the observation $o \in O$, the SU updates its belief $p_{t}$ and will also gain a certain reward. Let $\pi$ be the policy that maps the SU's belief $p_{t}$ to the action space $a \in A$ at each time $t$. Define the value function $U\left(p_{t}, t\right)$ as the maximum expected total reward at time $t$ when the current belief is $p_{t}$. This function specifies the performance of the optimal policy $\pi^{*}$, starting from belief $p_{t}$. Based on Bellman equation, we have the following:

$$
U\left(p_{t}, t\right)=\max \left\{U_{T R}\left(p_{t}, t\right), U_{T S}\left(p_{t}, t\right), U_{S O}\left(p_{t}, t\right), U_{C S}\left(p_{t}, t\right)\right\}
$$

where $U_{T R}\left(p_{t}, t\right), U_{T S}\left(p_{t}, t\right), U_{S O}\left(p_{t}, t\right)$, and $U_{C S}\left(p_{t}, t\right)$ are the expected total rewards if the SU decides to operate in the TR, 
TS, SO, and CS modes, respectively, at time $t$ and then follows the optimal policy $\pi^{*}$

\section{B. Reward Function}

In this section, we formulate the SU utility for various actions. Define the immediate and expected future reward that the SU gains from taking action $i$ as $R_{i}^{(M)}$ ( $M$ for myopic) and $R_{i}^{(L)}$ ( $L$ for long-term), respectively. The probability that the $i$ th SU observes outcome $o$ is denoted by $w_{o}^{(i)}$. The updated belief probability for outcome $o$ at node $i$ is denoted by $\mathcal{E}_{o}^{(i)}$. Define $q_{t}^{(T)}$ as the probability that the PU will remain idle during the transmission period $T$, given that the PU is idle at time $t$. Similarly, define $q_{t}^{(S)}(i)$ as the probability that the PU will remain idle during $T_{S i}, i=1,2, \ldots, m$, given that the PU is idle at the start of this sensing duration. These two quantities can be expressed as follows:

$$
\begin{gathered}
q_{t}^{(T)}=\frac{1-F_{X}(t+T)}{1-F_{X}(t)} \\
q_{t}^{(S)}(i)=\frac{1-F_{X}\left(t+\sum_{j=1}^{i} T_{S j}\right)}{1-F_{X}\left(t+\sum_{j=1}^{i-1} T_{S j}\right)} .
\end{gathered}
$$

where $F_{X}(t)$ is the CDF of random variable $X$ evaluated at point $t$.

Next, we derive the reward function for various SU modes.

1) TR mode: The myopic reward for the SU link, consisting of nodes $a$ and $b$, under the TR mode can be formulated as follows:

$$
R_{T R}^{(M)}=\sum_{i \in\{a, b\}} w_{D}^{(i)} T \log \left(1+S N R_{T R}^{(i)}\right) .
$$

where $w_{D}^{(i)}$ is the probability that the $i$ th SU has successfully decoded the received message, and $S N R_{T R}^{(i)}$ is the SNR in the TR mode at node $i$, which is given by:

$$
S N R_{T R}^{(i)}=\frac{P_{j}\left|h_{j i}\right|^{2}}{\sigma_{i}^{2}+\chi_{i}^{2} P_{i}\left|h_{i i}\right|^{2}} .
$$

In (26), $h_{i i}$ is the gain of the self-interfering channel at node $i$.

Since the two communicating SUs may experience different channel conditions, the ability to receive data differ from one node to another. Although, we assume that the PU signal affects both SUs equally, the interference level may differ from one node to another because of other interference sources. Hence, a successful decoding process at one node does not imply that the other node will be able to decode its packet. Also, the SU might get an ACK although the PU is ON, due to deep channel fading between the primary transmitter and the secondary receiver. All of these features are captured in the following two probabilities, which may differ from one SU to another [15]:

$\delta_{0}^{(i)}$ : probability that the $i t h$ secondary transmitter receives a NACK although the PU is OFF.

$\delta_{1}^{(i)}$ : probability that the $i t h$ secondary transmitter receives a NACK given that the PU is $\mathrm{ON}$.
When the ACK/NACK reflects only whether a collision occurs with the PU or not, we have $\delta_{0}^{(i)}=0$ and $\delta_{1}^{(i)}=1$.

The probability that the $i$ th $\mathrm{SU}, i=a, b$ successfully decode the received message is as follows:

$$
w_{D}^{(i)}=p_{t} q_{t}^{(T)}\left(1-\delta_{0}^{\hat{i}}\right)+\left(1-p_{t} q_{t}^{(T)}\right)\left(1-\delta_{1}^{\hat{i}}\right) .
$$

where $\hat{i}$ denotes the peer node of SU node $i$ (i.e., for link $(a, b)$, if $i=a$, then $\hat{i}=b$ and vice versa).

Using Bayes' rule, the probability that the PU is idle after $T$ given that the $i$ th $\mathrm{SU}$ successfully decode the received message (i.e., the belief update) is:

$$
\mathcal{E}_{D}^{(i)}=\left[p_{t} q_{t}^{(T)}\left(1-\delta_{0}^{\hat{i}}\right)\right] / w_{D}^{(i)}
$$

Similarly, the probability that the $i$ th SU failed to decode the received message and the belief update in that case can be written, respectively, as follows:

$$
\begin{gathered}
w_{U}^{(i)}=p_{t} q_{t}^{(T)} \delta_{0}^{\hat{i}}+\left(1-p_{t} q_{t}^{(T)}\right) \delta_{1}^{\hat{i}} \\
\mathcal{E}_{U}^{(i)}=\left[p_{t} q_{t}^{(T)} \delta_{0}^{\hat{i}}\right] / w_{U}^{(i)} .
\end{gathered}
$$

Since we assume that transmission errors in ACK/NACK are negligible, the probability of receiving an ACK/NACK at node $i$ is the same as the probability that its peer node succeed/fail in decoding the message. This is also applied to the belief update for the corresponding cases. Hence, $w_{A}^{(i)}, \mathcal{E}_{A}^{(i)}, w_{N}^{(i)}$, and $\mathcal{E}_{N}^{(i)}$ will be formulated similarly as $w_{D}^{(i)}, \mathcal{E}_{D}^{(i)}, w_{U}^{(i)}$, and $\mathcal{E}_{U}^{(i)}$, respectively.

There are four possible outcomes for the TR mode. An SU may receive an ACK for correct transmission and be able to successfully decode the received message, or the SU may get an ACK and an undecoded message. The other two outcomes of the TR mode is to either get a NACK and a decoded message, or a NACK and an undecoded message. Hence, the expected future reward for an SU link obtained at the $i$ th SU can be formulated as follows:

$$
R_{T R}^{(L)}=\sum_{\substack{k=\{A, N\} \\ l=\{D, U\}}} w_{k}^{(i)} w_{l}^{(i)} U\left(\mathcal{E}_{k}^{(i)} \mathcal{E}_{l}^{(i)}, t+T\right)
$$

where the summation over the two indices $(k, l)$ can be calculated by considering the four possible combinations $(A, D),(A, U),(N, D),(N, U)$.

Finally,

$$
U_{T R}\left(p_{t}, t\right)=R_{T R}^{(M)}+\eta R_{T R}^{(L)}
$$

where $\eta \in[0,1]$ is the discount factor, which determines how far you take the future reward into consideration while formulating the secondary utilities. At $\eta=0$, the SU only cares for the immediate reward. The final belief $p_{t+T}$ will be the multiplication of the two updates $\mathcal{E}_{\mathrm{O}_{1}}^{(i)} \mathcal{E}_{\mathrm{O}_{2}}^{(i)}$, where $O_{1} \in\{A, N\}$, and $O_{2} \in\{D, U\}$.

2) TS mode: The myopic reward of the TS mode is different from that of the TR mode because the SU is monitoring the spectrum while transmission. Hence, the SU could abort transmission if a busy outcome is observed after any 
$T_{S j}, j=1,2, \ldots, m$. Therefore, the myopic reward in the TS mode, assuming that $\mathrm{SU} i$ is transmitting to $\mathrm{SU} j$ will be formulated as follows:

$$
R_{T S}^{(M)}=\prod_{l=1}^{m} w_{F}^{(i)}(l) w_{A}^{(i)} T \log \left(1+S N R_{T S}^{(j)}\right)
$$

where the SNR in the TS mode at node $j$ is given by:

$$
S N R_{T S}^{(j)}=\frac{P_{i}\left|h_{i j}\right|^{2}}{\sigma_{j}^{2}} .
$$

$\prod_{l=1}^{m} w_{F}^{(i)}(l) w_{A}^{(i)}$ is the probability of successful transmission in the TS mode, which has two conditions. First, SU $i$ gets a free outcome after each and every sensing period of the $m$ sensing durations. This probability is denoted by $\prod_{l=1}^{m} w_{F}^{(i)}(l)$. Second, SU $i$ receives an ACK from $\mathrm{SU} j$ at the end of $T$, which is denoted by probability $w_{A}^{(i)}$. Define $\mathbb{P}_{f}=\left[\begin{array}{llll}P_{f, 1} & P_{f, 2} & \ldots & P_{f, m}\end{array}\right]$ and $\mathbb{P}_{d}=\left[\begin{array}{llll}P_{d, 1} & P_{d, 2} & \ldots & P_{d, m}\end{array}\right]$ as $m$-dimensional vectors that represent the false-alarm and detection probabilities, respectively, for the $m$ FD sensing periods in the TS mode.

The probability of getting ACK/NACK from the transmission process and the belief update in the corresponding cases are the same as that of the TR mode. The sensing process has also two outcomes, either free or busy. We assume that if the PU is sensed free/busy at time $t$ at one end of the SU link, then the other SU will experience the same situation. Also, if the PU is sensed busy at any sensing period, this yields a failure communication and the SU should abort the TS mode.

Hence, the probability that the $i$ th $\mathrm{SU}, i=a, b$ gets a free outcome after $T_{S 1}$ can be expressed as follows.

$w_{F}^{(i)}(1)=p_{t} q_{t}^{(S)}(1)\left(1-P_{f, 1}\right)+\left(1-p_{t} q_{t}^{(S)}(1)\right)\left(1-P_{d, 1}\right)$.

Similarly, the probability that the $i$ th SU gets a free outcome after $T_{S j}, j=2,3, \ldots, m$ given that it got a free outcome at $T_{S(j-1)}$ is as follows:

$$
w_{F}^{(i)}(j)=q_{t}^{(S)}(j)\left(1-P_{f, j}\right)+\left(1-q_{t}^{(S)}(j)\right)\left(1-P_{d, j}\right) .
$$

The belief update after $T_{S 1}$ and $T_{S j}, j=2,3, \ldots, m$ in the case of a free outcome can be written, respectively, as follows:

$$
\begin{gathered}
\mathcal{E}_{F}^{(i)}(1)=\left[p_{t} q_{t}^{(S)}(1)\left(1-P_{f, 1}\right)\right] / w_{F}^{(i)}(1) \\
\mathcal{E}_{F}^{(i)}(j)=\left[q_{t}^{(S)}(j)\left(1-P_{f, j}\right)\right] / w_{F}^{(i)}(j) .
\end{gathered}
$$

Similarly, the probability that the $i$ th $\mathrm{SU}, i=a, b$ gets a busy outcome after $T_{S 1}$ is:

$$
w_{B}^{(i)}(1)=p_{t} q_{t}^{(S)}(1) P_{f, 1}+\left(1-p_{t} q_{t}^{(S)}(1)\right) P_{d, 1} .
$$

Generally, the probability of getting a busy outcome after $T_{S j}, j=2,3, \ldots, m$ given that it got a free outcome at $T_{S(j-1)}$ is as follows:

$$
w_{B}^{(i)}(j)=q_{t}^{(S)}(j) P_{f, j}+\left(1-q_{t}^{(S)}(j)\right) P_{d, j} .
$$

The belief update after $T_{S 1}$ and $T_{S j}, j=2,3, \ldots, m$ in the case of a busy outcome can be written, respectively, as follows:

$$
\begin{aligned}
& \mathcal{E}_{B}^{(i)}(1)=\left[p_{t} q_{t}^{(S)}(1) P_{f, 1}\right] / w_{B}^{(i)}(1) \\
& \mathcal{E}_{B}^{(i)}(j)=\left[q_{t}^{(S)}(j) P_{f, j}\right] / w_{B}^{(i)}(j) .
\end{aligned}
$$

TS mode is different from other modes as the SU may not continue till the end of the transmission duration. This happens if the SU gets a busy outcome at the end of any sensing duration $T_{S i}, i=1,2 \ldots, m$. However, if the SU gets a free outcome after every sensing period $T_{S i}, i=1,2 \ldots, m$, there are two possible outcomes. The SU may get a Free and ACK for correct reception or Free and NACK for incorrect reception. Putting all together, the expected future reward in the TS mode can be formulated as follows:

$$
\begin{aligned}
R_{T S}^{(L)} & =\sum_{j=1}^{m} w_{B}^{(i)}(j) \prod_{l=1}^{j-1} w_{F}^{(i)}(l) U\left(\mathcal{E}_{B}^{(i)}(j) \prod_{l=1}^{j-1} \mathcal{E}_{F}^{(i)}(l), t+\sum_{l=1}^{j} T_{S l}\right) \\
& +\sum_{k=\{A, N\}} w_{k}^{(i)} \prod_{l=1}^{m} w_{F}^{(i)}(l) U\left(\mathcal{E}_{k}^{(i)} \prod_{l=1}^{m} \mathcal{E}_{F}^{(i)}(l), t+T\right)
\end{aligned}
$$

Finally,

$$
U_{T S}\left(p_{t}, t\right)=R_{T S}^{(M)}+\eta R_{T S}^{(L)}
$$

3) $S O$ mode: The immediate reward $R_{S O}^{(M)}$ in the SO mode will be zero as no transmission takes place. The outcome of the sensing process is either free or busy. The probability of getting a free/busy outcome and the belief update in each case can be formulated similarly as the sensing part of the TS mode taking into consideration that the SO mode consists of a single sensing period $T_{S}$. Hence, the expected future reward in the $\mathrm{SO}$ mode, assuming that $\mathrm{SU} i$ is sensing the spectrum, can be expressed as follows:

$$
R_{S O}^{(L)}=\sum_{k=\{F, B\}} w_{k}^{(i)} U\left(\mathcal{E}_{k}^{(i)}, t+T_{S}\right)
$$

where $w_{F}^{(i)}$ and $w_{B}^{(i)}$ are the probabilities of getting a free and busy outcomes, respectively, after $T_{S} \cdot \mathcal{E}_{F}^{(i)}$ and $\mathcal{E}_{B}^{(i)}$ are the corresponding belief updates. Note that while formulating these quantities, we should include the false-alarm and detection probabilities in the HD case (i.e., $\tilde{P}_{f}$ and $\tilde{P}_{d}$ ). Also, the probability that the PU will remain idle during the sensing period $T_{S}$, given that the PU is idle at time $t$ will be expressed as follows:

$$
q_{t}^{(S)}=\frac{1-F_{X}\left(t+T_{S}\right)}{1-F_{X}(t)}
$$

Hence we can write the maximum expected utility that the SU gains from sensing the spectrum as:

$$
U_{S O}\left(p_{t}, t\right)=\eta R_{S O}^{(L)} .
$$

4) CS mode: The SU might choose to switch to another frequency channel (where no information about the PU state is available) and carry out spectrum sensing, if the probability that the PU returns is very high. The analysis for this operation mode is the same as that of SO mode, except for the belief $p_{t}$ because the belief in the new channel $\hat{p}_{t}$ will be the probability 
that the PU is idle at time $t$ given that no previous information is available, which can be written generally as follows:

$$
\hat{p}_{t}=\bar{X} /(\bar{X}+\bar{Y}) \text {. }
$$

The maximum expected utility for the CS mode is as follows:

$$
U_{C S}\left(\hat{p}_{t}, t\right)=\eta R_{C S}^{(L)}
$$

where

$$
R_{C S}^{(L)}=\sum_{k=\{F, B\}} \hat{w}_{k}^{(i)} U\left(\hat{\mathcal{E}}_{k}^{(i)}, t+T_{S}\right)
$$

where $\hat{w}_{F}^{(i)}, \hat{w}_{B}^{(i)}, \hat{\mathcal{E}}_{F}^{(i)}$ and $\hat{\mathcal{E}}_{B}^{(i)}$ are formulated similarly as $w_{F}^{(i)}, w_{B}^{(i)}, \mathcal{E}_{F}^{(i)}$ and $\mathcal{E}_{B}^{(i)}$, respectively, after replacing $p_{t}$ by $\hat{p}_{t}$.

In our online technical report [25], we discuss the convexity and other properties of the SU's utilities $U_{T R}\left(p_{t}, t\right), U_{T S}\left(p_{t}, t\right), U_{S O}\left(p_{t}, t\right)$, and $U_{C S}\left(\hat{p}_{t}, t\right)$ with respect to the belief. We also discuss the convexity of $U\left(p_{t}, t\right)$ in the belief and prove that it increases with $p$ for a given $t$ using backward induction.

\section{Optimal Policy}

After formulating the SU utilities in the four possible actions and adding a constraint on the collision probability with the PU, our problem can be formulated as follows:

$$
\begin{array}{ll}
\underset{\pi}{\operatorname{maximize}} & U\left(p_{t}, t\right) \\
\text { subject to } & P_{i} \leq P_{i}^{*} \quad i \in\{T R, T S\}
\end{array}
$$

where $P_{i}, i \in\{T R, T S\}$ is the PU collision probability in the TR and TS modes, respectively and $P_{i}^{*}$ is the threshold PU collision probability. $P_{T R}$ and $P_{T S}$ can formulated as follows:

$$
\begin{gathered}
P_{T R}=\left(1-p_{t}\right)+p_{t}\left(1-q_{t}^{(T)}\right)=1-p_{t} q_{t}^{(T)} \\
P_{T S}=p_{t} \sum_{i=1}^{m}\left\{\prod_{j=1}^{i-1}\left(1-P_{f, j}\right)\left(1-q_{t}^{(S)}(i)\right)\right\}+\left(1-p_{t}\right) .
\end{gathered}
$$

For certain probability distributions for the PU idle period as Gaussian distribution, uniform distribution, and Rayleigh distribution (as an example), $q_{t}^{(T)}$ will approach zero at large values of $t$. This means that the probability that the PU returns to utilize the channel increases with $t$, which is very intuitive for this type of distributions. To be able to derive our optimal threshold-based policy, we define a technical condition similar to the approach followed in [15]. This technical condition states that for all $t>t^{*}$, the SU should not transmit any data (i.e. should not operate in either TR, or TS modes) as the collision probability constraint will not be satisfied and hence, zero reward will be gained even at $p_{t}=1$. This threshold time $t^{*}$ is defined as the minimum time where the PU collision constraint is not satisfied. Hence, $U(1, t)=0, \forall t>t^{*}$.

$$
t^{*}=\min \left\{t: q_{t}^{(T)}<1-P_{T R}^{*}\right\} .
$$

Theorem 1: The optimal policy for the SU can be written as follows:

$$
\pi^{*}\left(p_{t}\right)= \begin{cases}C S, & p_{t}<\beta_{c} \\ S O, & \beta_{c} \leq p_{t}<\beta_{s} \\ T S, & \beta_{s}<p_{t}<\beta_{t} \\ T R, & p_{t} \geq \beta_{t}\end{cases}
$$

The proof of the theorem is omitted due to space limit and can be reached in our technical report [25]. The above theorem states that the SU should utilize the opportunity of having a high belief that the PU is idle and operate in the TR mode if $p_{t} \geq \beta_{t}$, where $\beta_{t}$ is the transmission-reception threshold. In that case the SU will dramatically increase the throughput by transmitting and receiving data simultaneously over the same channel. If the belief decreases and falls in the following range $\beta_{s}<p_{t}<\beta_{t}$, the SU should monitor the spectrum while transmitting (i.e., operate in the TS mode) as the probability that the SU returns is now relatively high. $\beta_{s}$ is called the sensing threshold. In that case, the SU still getting some throughput (lower than TR mode), however a lower collision probability is achieved. The SU should stop transmitting, and carry out HD sensing (i.e., SO mode) if $p_{t}$ is relatively low (i.e., $\beta_{c} \leq p_{t}<\beta_{s}$ ) because in that case the probability that the PU returns to the channel is too high and the PU collision constraint will not be satisfied. Hence, a better sensing quality and a temporarily channel vacation is required. $\beta_{c}$ is called the channel switching threshold. At very low belief values $p_{t}<\beta_{c}$, where the PU is most likely to return to use the channel, the SU should take the CS action. This happens when the probability that the PU is idle in a new channel (where no information is available) is higher than the current belief.

To solve our problem, we have to find the threshold time $t^{*}$, where our condition is satisfied and then apply backward induction to find the thresholds $\beta_{c}, \beta_{s}, \beta_{t}$ and the maximum utility for the SU $U(p, t)$ for different values of $p$ and $t$.

\section{Discussion of the optimal policy}

In this section, we will highlight some important features of our optimal policy that should be considered in our FD DSA network. Until this point of discussion, we assumed that SUs always have data to transmit. That is, if the optimal policy recommends the TR mode, then SUs will carry out simultaneous transmission and reception over the same frequency. But, what if one of the nodes does not have data to its peer at this instant? This motivates the refinement of our adaptive strategy to account for SU's traffic flow. Before doing that, let us first discuss how we define master/slave nodes, and data/control phases in the following two items:

- Master and slave nodes: A master node is a designation given to any SU device that executes the optimal adaptive decision strategy. The master node is the one that takes a final decision about the operation mode, whether receiving data while transmitting, sensing the spectrum while transmitting, switching to another channel, or solely sensing the spectrum. The slave node is the SU device that is receiving orders from the master node with regard to starting, continuing, or stopping transmission. This is done through control packets. In the present design, the node that initiates the communication is the master node, and the other 
one is the slave node. However, these roles may change over time, depending on which node has traffic to send (i.e., the traffic directionality).

- Data and Control Phases: This concept is related to the organizing protocol used between SUs, equipped with SIS/FD capabilities, to communicate with each other. Once a channel is thought to be idle by the initiating $\mathrm{SU}$, the time frame will be divided into two alternating phases: data phase and control phase. SUs will start communicating together in the data phase where data packets only are exchanged. Also, spectrum sensing is executed in the data phases as well. After that, a control phase is established between the two nodes, which has two goals. First, this control phase is used to confirm the correct reception of packets transmitted in the previous data phase in both directions, if applicable. Second, the control phase is used by the master node to trigger the slave node to start, continue, or stop transmission while receiving. If the two nodes switch to another channel, the data and control phases will be terminated (until finding a free channel) and the sensing durations can be optimized separately, without the data and control phases restrictions.

The four possible modes (TR, TS, SO, and CS) represent a single SU's perspective. However, the link's operation is determined by the mode of operation at the two communicating nodes. Hence, these modes of operation should be written as follows: TR-TR, TS-R (or R-TS), SO, and CS. For instance, if an SU is transmitting and receiving data simultaneously, the other SU will also be transmitting and receiving data, which is indicated by the TR-TR mode. However, if an SU is transmitting and sensing, its peer will be only in the reception mode, which defines the TS-R mode, where $\{\mathrm{R}\}$ stands for reception. The threshold-based structure discussed so far has to be adjusted with the SUs traffic load. For instance, assume that the optimal strategy recommends the master SU to operate in the TR mode, while the slave node does not have data to transmit. In this case, the master SU node can operate in the TS mode while the slave SU can only receive data. To implement this, the header of the transmitted packet should be modified to include a bit (name it the "more packets" (MP) bit) that tells whether the transmitter has more packets in its queue or not. The two communicating SUs can utilize the MP bit to determine the final decision.

Another crucial point in FD DSA networks to be considered in our design is the FCC requirements. Consider the scenario where SUs are operating in the idle PU period using the TR mode. In the case of good channel conditions, both nodes will keep ACKing their packets, updating their beliefs, and will not switch to any other mode unless they collide with the PU (in that case they will get NACKs), or when $t>t^{*}$. To avoid this blind communication without monitoring the PU channel, SUs should periodically switch to any of the sensing modes (TS or SO), if they violate the FCC requirements discussed next. The FCC imposes rules for operating opportunistic wireless networks. One of these rules is the periodic sensing interval, which means that any channel used by an SU has to be sensed every $T_{r e q}$ seconds to check for the PU activity. The SU has to vacate the channel quickly if a PU activity is detected. Hence, this part of the decision

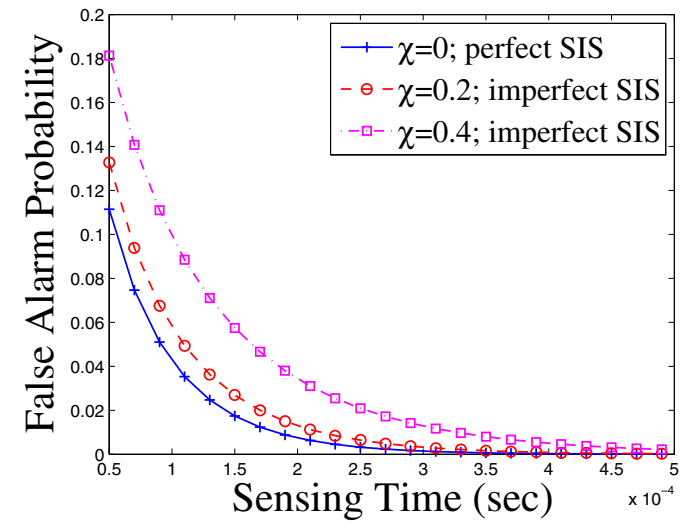

Fig. 4. False-alarm probability vs. sensing time for FD sensing at different values of $\chi$.

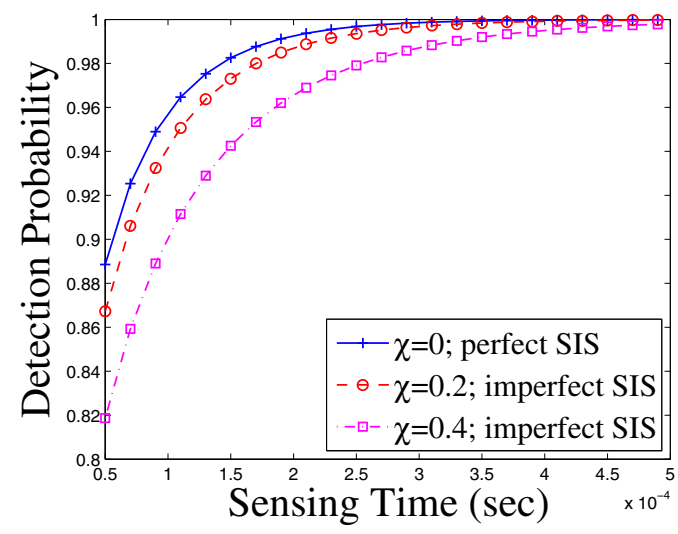

Fig. 5. Detection probability vs. sensing time for FD sensing at different values of $\chi$.

strategy states that each SU link has to maintain a maximum duration of $T_{r e q}$ seconds between sensing periods, whether it was operating in the SO or TS modes.

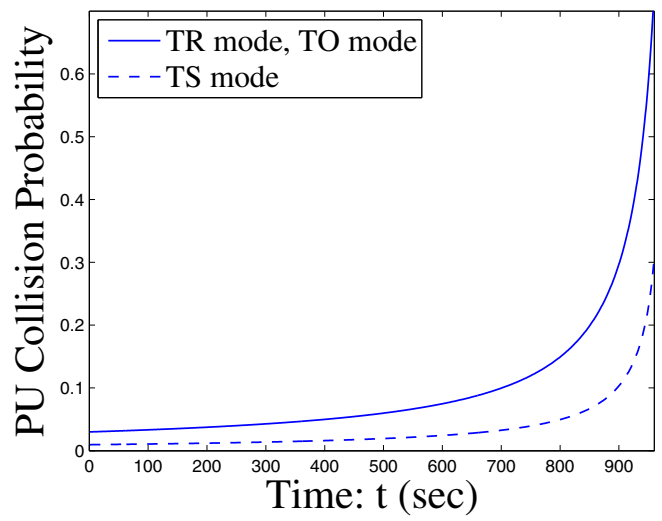

Fig. 6. PU collision probability vs. $t$ for TR, TS, and TO modes at $p=1$ and $P_{f}=0.1$. 


\section{Numerical Results}

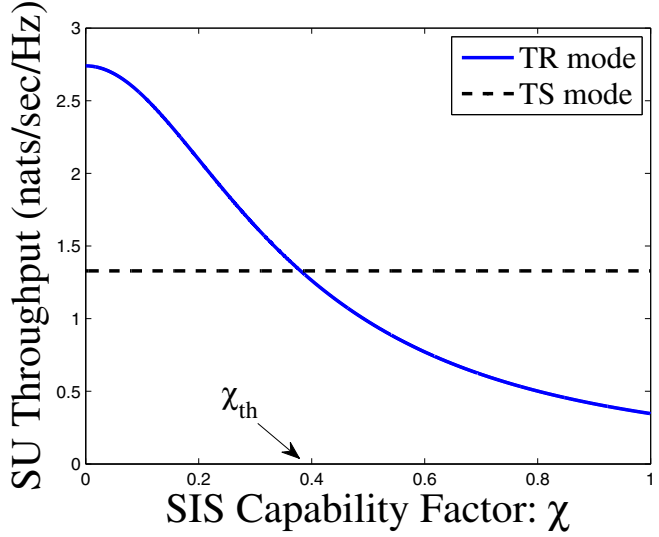

Fig. 7. SU's throughput vs. $\chi$ for TR and TS modes at $p=1, P_{f}=0$, $P_{d}=1, \sigma_{s}^{2}=15$ and $S N R^{(H D)}=5 d B$.

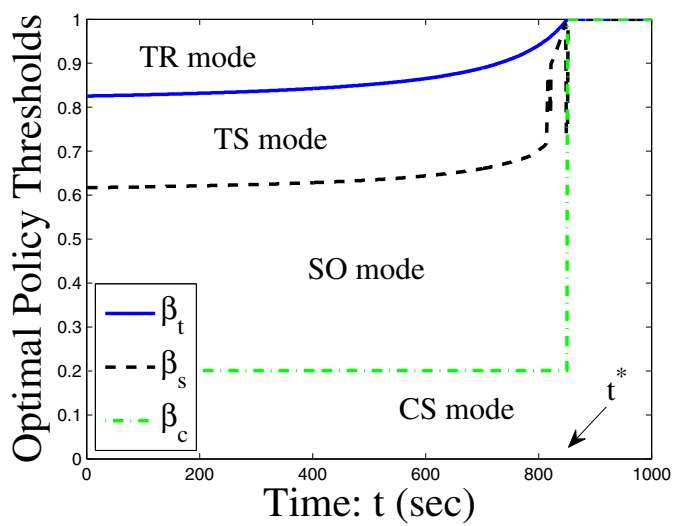

Fig. 8. Optimal policy thresholds vs. $t$. The decision region is divided into four parts: TR, TS, SO, and CS.

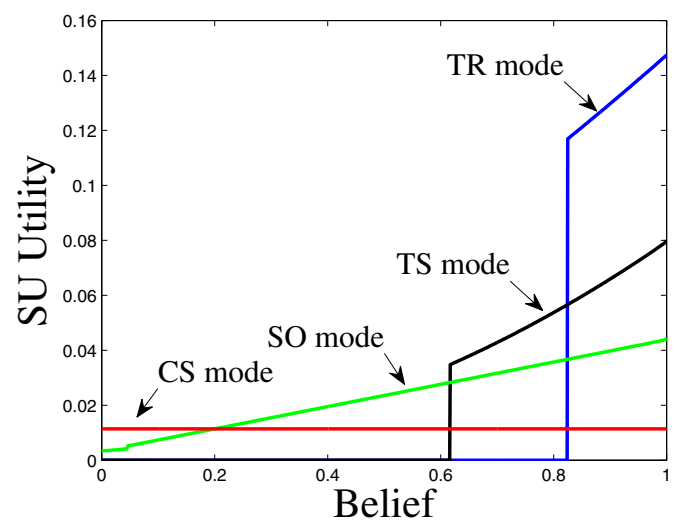

Fig. 9. SU utilities in the TR, TS, SO, and CS modes vs. the belief at $t=0$. The final SU utility is the maximum of these four utilities.
We use the following parameters unless otherwise is mentioned. The sampling frequency and the SU signal power are $f_{S}=6 \mathrm{MHz}$ and $\sigma_{s}^{2}=5$, respectively and $S N R^{(H D)}=$ $-20 d B$. For evaluating $P_{f}$ and $P_{d}$, we consider a complex Gaussian primary signal with $\alpha=2$. The PU idle period is uniformly distributed in the range $[0,1000]$. We also set $T_{S}=1, m=30, T=30, \delta_{0}=0.01$, and $\delta_{1}=0.99$.

\section{A. Performance Metrics}

1) False-Alarm And Detection Probabilities: The impact of the residual self-interference signal on $P_{f}$ and $P_{d}$ for waveform-based sensing is shown in Figures 4 and 5, respectively. As $\chi$ increases the performance of the waveform-based sensing gets worse (i.e., $P_{f}$ increases and $P_{d}$ decreases) due to the increment in the residual self-interference. We also notice that $P_{f}$ and $P_{d}$ converges to HD sensing at perfect SIS. In imperfect sensing schemes, increasing the sensing duration improves the performance of the sensing technique. At low SNR regions, the SU needs around $20 \%$ increment in the sensing duration to achieve the same $P_{f}$ and $P_{d}$ (achieved for HD sensing) for $20 \%$ residual self-interference from the original SU signal and needs about $80 \%$ increment in $T_{S}$ for $\chi=0.4$ (i.e., for a residual self-interference signal that is $40 \%$ of the original transmitted signal).

2) $P U$ Collision Probability: The advantage of the TS mode over the TR mode is the lower collision probability. Figure 6 shows the variation of the PU collision probability for the TR, TS, and TO mode with $t$. As $t$ increases, the collision probability increase as the $\mathrm{PU}$ is more likely to return to utilize the channel. As shown in Figure 6, the SU can achieve a lower collision probability in the TS mode than that of the TR mode. This improvement may reach in certain scenarios up to $50 \%$ reduction in the collision probability.

3) SU Throughput: Figure 7 shows a comparison between the achievable SU's throughput in the TS mode (assuming long enough $\left.T_{S}\right)$ and the TR mode at different values of $\chi$. The SU's throughput in the TR mode decreases as $\chi$ increases due to residual self-interference. Hence, working in a FD fashion is not always optimal especially at high $\chi$ values. According to our simulation setup, the threshold SIS factor where the $\mathrm{SU}$ should switch to the TS mode is $\chi_{t h}=0.38$. Also, as $t$ increases the SU's throughput decreases due to the increment in the probability that PU returns.

\section{B. Transmission-Sensing-Reception Strategy}

We use backward induction to find the optimal thresholds and the maximum SU utility. We set $\eta=0.3, P_{T R}^{*}=0.2, P_{T S}^{*}=$ $0.4, P_{f}=0.01, P_{d}=0.99, S N R^{(H D)}=20 d B$, and $\bar{Y}=2000$.

The variation of $\beta_{t}, \beta_{s}$ and $\beta_{c}$ with $t$ is shown in Figure 8 , which shows the mechanism of our optimal policy. The SU should operate in TR mode as long as $p_{t} \geq \beta_{t}$, switch to TS mode when $\beta_{s}<p_{t}<\beta_{t}$, switch to SO mode when $\beta_{c} \leq p_{t}<\beta_{s}$, and finally switch to a new channel when $p_{t}<\beta_{c}$. The SU should also switch to a new channel if $t>t^{*}$ as the PU is more likely to return to utilize the channel, which justifies the convergence of $\beta_{t}, \beta_{s}$ and $\beta_{c}$ to 1 for $t>t^{*}$. Note that $\beta_{c}$ is constant (for $t<t^{*}$ ) because it depends on the 
channel availability, when no information is known. Hence, $\beta_{c}=500 / 2500=0.2$ according to our setup.

Figure 9 shows the variation of the maximum SU utilities for the TR, TS, SO, and CS modes with $p_{t}$. The final SU utility is the maximum of these four utilities. Note that the utility in the CS mode is constant with $p$ because it is independent of the SU belief in the currently used channel. The abrupt reduction for the SU utilities in the TR and TS modes is due to the violation of the PU collision probability constraints.

\section{CONCLUSIONS}

We consider a novel application of FD communications in DSA networks, where we analyzed the performance metrics of the TR and TS modes, namely the throughput and collision probability. We determined the optimal switching policy of SUs, equipped with SIS/FD capabilities, that maximizes the SU's utility subject to a constraint on the PU collision probability. To enable the TS mode, we analyzed the waveform-based sensing in the case of imperfect SIS, and derived the falsealarm and detection probabilities. Using our adaptive strategy, the SU can achieve about $50 \%$ reduction in the collision probability and double the throughput comparing to the HD case. Finally, an optimal threshold-based strategy is obtained, which depends on the SU's belief regarding the idleness of the PU. Our results indicate that the SU should operate in the TR mode if it has a high belief that the PU is idle. As this belief decreases, the SU should adaptively switch to the TS mode to monitor any change in the PU activity while transmitting. At very low belief values, where the PU is more likely to be active, the SU should switch to another channel.

One possible direction of future work is to address how SUs will negotiate in the control phase to determine the final action given that both nodes may have different traffic flows. Another direction for future work could be to optimize the parallel sensing durations of the TS mode taking into account the tradeoff between sensing efficiency and the timeliness in detecting PU activity.

\section{ACKNOWLEDGMENTS}

This research was supported in part by the National Science Foundation (grants numbers IIP-1265960 and CNS-1016943). Any opinions, findings, conclusions, or recommendations expressed in this paper are those of the authors and do not necessarily reflect the views of the National Science Foundation.

\section{REFERENCES}

[1] J. I. Choi, M. Jain, K. Srinivasan, P. Levis, and S. Katti, "Achieving single channel, full duplex wireless communication," in Proc. of the ACM Mobicom'10 Conf., Sep. 2010, pp. 1-12.

[2] M. Jain, J. I. Choi, T. Kim, D. Bharadia, S. Seth, K. Srinivasan, P. Levis, S. Katti, and P. Sinha, "Practical, real-time, full duplex wireless," in Proc. of the ACM Mobicom'11 Conf., Sep. 2011, pp. 301-312.

[3] M. Duarte and A. Sabharwal, "Full-duplex wireless communications using off-the-shelf radios: Feasibility and first results," in Proc. of the ASILOMAR'10 Conf., Nov. 2010, pp. 1558-1562.

[4] E. Everett, M. Duarte, C. Dick, and A. Sabharwal, "Empowering fullduplex wireless communication by exploiting directional diversity," in Proc. of the ASILOMAR'11 Conf., 2011, pp. 2002-2006.
[5] B. Radunovic, D. Gunawardena, P. Key, A. Proutiere, N. Singh, V. Balan, and G. Dejean, "Rethinking indoor wireless mesh design: Low power, low frequency, full-duplex," in Proc. of the Fifth IEEE Workshop on Wireless Mesh Networks, 2010.

[6] D. Bharadia, E. McMilin, and S. Katti, "Full duplex radios," in Proc. of the ACM SIGCOMM'13 Conf., Aug. 2013.

[7] S. Barghi, A. Khojastepour, K. Sundaresan, and S. Rangarajan, "Characterizing the throughput gain of single cell MIMO wireless systems with full duplex radios," in Proc. of the WiOpt'12 Conf., 2012, pp. 68-74.

[8] D. Ng, E. Lo, and R. Schober, "Dynamic resource allocation in MIMO-OFDMA systems with full-duplex and hybrid relaying," IEEE Transactions on Communications, vol. 60, no. 5, pp. 1291-1304, 2012.

[9] B. Day, A. Margetts, D. Bliss, and P. Schniter, "Full-duplex bidirectional MIMO: Achievable rates under limited dynamic range," IEEE Transactions on Signal Processing, vol. 60, no. 7, pp. 3702-3713, 2012.

[10] E. Aryafar, M. A. Khojastepour, K. Sundaresan, S. Rangarajan, and M. Chiang, "MIDU: Enabling MIMO full duplex," in Proc. of the ACM Mobicom'12 Conf., 2012, pp. 257-268.

[11] W. Afifi and M. Krunz, "Exploiting self-interference suppression for improved spectrum awareness/efficiency in cognitive radio systems." in Proc. of the IEEE INFOCOM'13 Conf., 2013, pp. 1258-1266.

[12] W. Cheng, X. Zhang, and H. Zhang, "Full duplex spectrum sensing in non-time-slotted cognitive radio networks," in Proc. of the MILCOM'11 Conf., Nov. 2011.

[13] _ " "Optimal dynamic power control for full-duplex bidirectionalchannel based wireless networks," in Proc. of the IEEE INFOCOM'13 Conf., Turin, Italy, Apr. 2013.

[14] Q. Zhao and J. Ye, "Quickest detection in multiple on-off processes," IEEE Transactions on Signal Processing, vol. 58, no. 12, pp. 59946006, Dec. 2010.

[15] S. Huang, X. Liu, and Z. Ding, "Optimal sensing-transmission structure for dynamic spectrum access," in Proc. of the IEEE INFOCOM'09 Conf., April 2009, pp. 2295-2303.

[16] _ - "Short paper: On optimal sensing and transmission strategies for dynamic spectrum access," in Proc. of the IEEE DySPAN'08 Conf., Oct. 2008, pp. 1-5.

[17] W. Afifi, A. Sultan, and M. Nafie, "Adaptive sensing and transmission durations for cognitive radios," in Proc. of the IEEE DySPAN'11 Conf., 2011, pp. 380-388.

[18] D. Willkomm, S. Machiraju, J. Bolot, and A. Wolisz, "Primary users in cellular networks: A large-scale measurement study," in Proc. of the IEEE DySPAN'08 Conf., Oct. 2008, pp. 1 -11.

[19] H. Kim and K. Shin, "Efficient discovery of spectrum opportunities with MAC-layer sensing in cognitive radio networks," IEEE Transactions on Mobile Computing, vol. 7, no. 5, pp. 533-545, May 2008.

[20] Y. Yuan, P. Bahl, R. Chandra, T. Moscibroda, and Y. Wu, "Allocating dynamic time-spectrum blocks in cognitive radio networks," in Proc. of the ACM MobiHoc'07 Conf., Sep. 2007, pp. 130-139.

[21] T. Shu and M. Krunz, "Throughput-efficient sequential channel sensing and probing in cognitive radio networks under sensing errors," in Proc. of the ACM Mobicom'09 Conf., Sep. 2009, pp. 37-48.

[22] H. Jiang, L. Lai, R. Fan, and H. Poor, "Optimal selection of channel sensing order in cognitive radio," IEEE Transactions on Wireless Communications, vol. 8, no. 1, pp. 297-307, 2009.

[23] H. Tang, "Some physical layer issues of wide-band cognitive radio systems," in Proc. of the IEEE DySPAN'05 Conf., 2005, pp. 151-159.

[24] T. Yucek and H. Arslan, "A survey of spectrum sensing algorithms for cognitive radio applications," IEEE Communications Surveys and Tutorials, vol. 11, no. 1, pp. 116-130, 2009.

[25] W. Afifi and M. Krunz, "Optimal transmission-sensingreception strategies for full-duplex dynamic spectrum access," University of Arizona, Department of ECE, TR-UA-ECE2013-4, Tech. Rep., November 2013. [Online]. Available: http://www2.engr.arizona.edu/ krunz/publications.htm

[26] R. D. Smallwood and E. J. Sondik, "The optimal control of partially observable markov processes over a finite horizon," Operations Research, vol. 21, no. 5, pp. 1071-1088, 1973. 